\title{
Spectrally extremal vertices, strong cospectrality and state transfer
}

\author{
Gabriel Coutinho* \\ Dept. Combinatorics and Optimization \\ University of Waterloo \\ Waterloo, ON, Canada \\ gcoutinho@uwaterloo.ca
}

Submitted: Feb 9, 2015; Accepted: Feb 23, 2016 ; Published: Mar 4, 2016

Mathematics Subject Classifications: 05C50, 05E30, 81P68

\begin{abstract}
In order to obtain perfect state transfer between two sites in a network of interacting qubits, their corresponding vertices in the underlying graph must satisfy a property called strong cospectrality. Here we determine the structure of graphs containing pairs of vertices which are strongly cospectral and satisfy a certain extremal property related to the spectrum of the graph. If the graph satisfies this property globally and is regular, we also show that the existence of a partition of the vertex set into pairs of vertices at maximum distance admitting perfect state transfer forces the graph to be distance-regular. Finally, we present some new examples of perfect state transfer in simple graphs constructed with our technology. In particular, for odd distances, we improve the known trade-off between the distance perfect state transfer occurs in simple graphs and the size of the graph.
\end{abstract}

Keywords: Graph theory, Quantum walks, Cospectral vertices

\section{Introduction}

Let $X$ be a simple undirected graph, and consider its 01-adjacency matrix $A=A(X)$. For $u \in V(X)$, we denote by $e_{u}$ the vector of the canonical basis corresponding to $u$ in the ordering of the rows of $A$. The matrix operator

$$
U(t)=\exp (\mathrm{i} t A)
$$

defined for every real $t \geqslant 0$, represents a continuous-time quantum walk on $X$. It was shown in [7] that the dynamics of quantum state transfer in a network of interacting

\footnotetext{
* Research partialy funded by the CAPES foundation, Ministry of Education, Brazil.
} 
qubits in the $X Y$-coupling model is determined by properties of $U(t)$. More specifically, we will say that $X$ admits perfect state transfer between vertices $u$ and $v$ at time $\tau$ if

$$
\left|e_{v}^{T} U(\tau) e_{u}\right|=1
$$

The problem of determining which graphs admit perfect state transfer has been studied recently in a good number of papers. For example, it was solved for paths and hypercubes (see [6]), circulant graphs (see [19]) and cubelike graphs (see [5]). The effect of certain graph operations was considered in [1] and in [2], and some recent surveys are found in [17] and [14].

The study of state transfer or more generally the study of continuous-time quantum walks on graphs can be divided into two aspects. The algebraic properties that must be satisfied by the graph, and the number theoretic properties that must be satisfied by the eigenvalues of the graph. In the case of perfect state transfer between vertices $u$ and $v$, the key algebraic property is that $u$ and $v$ must be strongly cospectral, that is, the projections of $e_{u}$ and $e_{v}$ in each eigenspace of the graph must be parallel with the same magnitude.

In this paper, we will examine this property in the context of graphs which are extremal with respect to the lower bound on the number of eigenvalues given by the diameter plus one. State transfer on such graphs was considered in [21]. Our approach is nevertheless more general and results in deeper consequences.

We will consider the extremal spectral property locally, and show that a pair of strongly cospectral extremal vertices at maximum distance must be singletons in a pseudo equitable distance partition. This will be our key intermediate step to show that if an extremal regular graph of diameter $d$ can be partitioned into pairs of vertices at distance $d$ such that perfect state transfer happens in each pair, then the graph is distance-regular. We will also characterize precisely which number theoretic conditions must be satisfied by the eigenvalues of the graph in order to achieve perfect state transfer between strongly cospectral vertices. In the context of distance-regular graphs, this will provide an alternate elementary proof of [8, Corollary 4.5].

\section{Preliminaries}

Let $X$ be a graph, $u, v \in V(X)$, and $A$ the adjacency matrix of $X$. We denote the spectral decomposition of $A$ into orthogonal projections by

$$
A=\sum_{r=0}^{d} \theta_{r} E_{r} .
$$

Vertices $u$ and $v$ are called cospectral if the adjacency matrices of $X \backslash u$ and $X \backslash v$ have the same spectrum.

Lemma 1 ([13], Chapter 4, Lemma 1.1). The following are equivalent.

(i) Vertices $u$ and $v$ are cospectral. 
(ii) For all $k \in \mathbb{Z}^{+}$,

$$
\left(A^{k}\right)_{u, u}=\left(A^{k}\right)_{v, v}
$$

(iii) For all $r=0, \ldots, d$,

$$
\left(E_{r}\right)_{u, u}=\left(E_{r}\right)_{v, v}
$$

Recall that we denote the characteristic vector of a vertex $w$ by $e_{w}$. Vertices $u$ and $v$ are strongly cospectral if and only if $E_{r} e_{u}= \pm E_{r} e_{v}$ for all $r=0, \ldots, d$. Thus strongly cospectral vertices must be cospectral.

We define the eigenvalue support of $u$ as the set of all eigenvalues $\theta_{r}$ such that $E_{r} e_{u} \neq 0$, and we denote it by $\Phi_{u}$. We say that the dual degree of $u$ is $d^{*}(u)=\left|\Phi_{u}\right|-1$. The eccentricity (or covering radius) of $u$, denoted by $\varepsilon_{u}$, is the maximum distance between $u$ and any other vertex of the graph.

The distance partition relative to $u \in V(X)$ is the partition of the vertex set of $X$ in which each class is formed by vertices at a fixed distance from $u$. A partition of the vertex set of a graph is called equitable if the number of edges between a vertex $v$ and a class $C$ of the partition depends only on the class containing $v$. The collection of all these numbers are the parameters of the equitable partition.

If $D$ is a non-singular diagonal matrix, we consider the matrix $\left(D^{-1} A D\right)$. This can be seen as the adjacency matrix of a weighted directed graph, where $\left(D^{-1} A D\right)_{u v}$ indicates the weight of the directed arc from $u$ to $v$. We will call a partition of $V(X)$ equitable with respect to $D$ if the sum of the weights in $\left(D^{-1} A D\right)$ from a vertex $v$ to a class $C$ of the partition depends only of the class containing $v$.

When $D$ is chosen to be the diagonal matrix whose entries are taken from the eigenvector of $A$ corresponding to the largest eigenvalue, an equitable partition with respect to $D$ will simply be called pseudo equitable. Note that for regular graphs, a partition is pseudo equitable if and only if it is equitable in the original sense.

A graph is called distance-regular if the distance partition relative to each vertex is equitable and the parameters do not depend on the vertex. A distance-regular graph of diameter $d$ is called antipodal if the relation defined on the vertex set by having two vertices related if they are at distance 0 or $d$ is an equivalence relation.

Theorem 2 ([16], Theorem 2.2). If $X$ is a regular graph such that the distance partition of every vertex is equitable, then $X$ is distance-regular.

The walk module of a vertex $u$ in a graph $X$ is the subspace

$$
W_{u}=\left\langle\left\{A^{i} e_{u}\right\}_{i \geqslant 0}\right\rangle \text {. }
$$

It follows that

$$
W_{u}=\left\langle\left\{E_{r} e_{u}\right\}_{\theta_{r} \in \Phi_{u}}\right\rangle
$$

and because the vectors $\left\{A^{i} e_{u}\right\}_{i=0}^{\varepsilon_{u}}$ are all independent, we have that

$$
\varepsilon_{u} \leqslant d^{*}(u) .
$$


(see for instance [3, Proposition 2.6]). If equality holds, we say that $u$ is a spectrally extremal vertex. The work of Fiol, Garriga and others provides a vast literature about graphs with spectrally extremal vertices and associated concepts (see for instance [10], [9], [18] and [20]). Here we will use the following result.

Theorem 3 ([11], Theorem 6.3). The distance partition relative to a vertex $u$ is pseudo equitable if and only if $u$ is spectrally extremal and there exists a polynomial $p(x)$ such that $p(A) e_{u}$ is a vector whose support consists of the vertices at distance $\varepsilon_{u}$ from $u$.

On the topic of perfect state transfer, we will use the following two results.

Theorem 4 ([15], Theorem 6.1). If $X$ admits perfect state transfer between $u$ and $v$, then either all eigenvalues in the eigenvalue support of $u$ are integers, or they are all of the form $\theta_{r}=\frac{1}{2}\left(a+b_{r} \sqrt{\Delta}\right)$ where $\Delta$ is a square-free integer and a and $b_{r}$ are integers for all $r$.

Lemma 5 ([14], Lemma 11.1). If $X$ admits perfect state transfer between $u$ and $v$, then $u$ and $v$ are strongly cospectral.

\section{$3 \quad$ Strong cospectrality on spectrally extremal vertices}

It turns out that when two strongly cospectral vertices are also spectrally extremal, each of them is a singleton in the distance partition of the other.

Lemma 6. Let $u, v \in V(X)$, with $g=d(u, v)$. Suppose $u$ is a spectrally extremal vertex. If $u$ and $v$ are strongly cospectral, then the following hold.

(i) If $d(u, w)=d(u, v)$, then $w=v$.

(ii) If $z \in V(X), \Phi_{z}=\Phi_{u}$ and if $d(z, w)=d(u, v)$ for some $w \in V(X)$, then $\left(A^{g}\right)_{z, w} \leqslant$ $\left(A^{g}\right)_{u, v}$. Equality occurs if and only if $z$ and $w$ are strongly cospectral.

Proof. Suppose $\Phi_{u}=\left\{\theta_{0}, \theta_{1}, \ldots, \theta_{d^{*}}\right\}$. For all $r \in\left\{0, \ldots, d^{*}\right\}$, let $\sigma_{r} \in\{+1,-1\}$ be such that

$$
E_{r} e_{v}=\sigma_{r} E_{r} e_{u}
$$

Let $p(x)$ be the polynomial of minimum degree satisfying $p\left(\theta_{r}\right)=\sigma_{r}$ for all $r$. Then it follows that

$$
p(A) e_{u}=e_{v} .
$$

Because $\varepsilon_{u}=d^{*}, p(A) e_{u}$ must be non-zero on the entries corresponding to vertices whose distance to $u$ is the degree of $p(x)$. Hence $\operatorname{deg} p(x)=g$, and $v$ is the unique vertex at distance $g$ from $u$.

To see (ii), first note that $\left\langle p(A) e_{z}, p(A) e_{z}\right\rangle=1$, so the absolute value of each entry in $p(A) e_{z}$ is at most 1 . Let $p(x)=a_{g} x^{g}+\cdots+a_{0}$. Then $p(A) e_{u}=e_{v}$ implies that

$$
a_{g}=\frac{1}{\left(A^{g}\right)_{u, v}}
$$


and thus

$$
1 \geqslant\left|p(A)_{z, w}\right|=a_{g}\left(A^{g}\right)_{z, w}=\frac{\left(A^{g}\right)_{z, w}}{\left(A^{g}\right)_{u, v}}
$$

We are also going to need the following characterization of strongly cospectral vertices.

Lemma 7. Let $u, v \in V(X)$. The following are equivalent.

(i) Vertices $u$ and $v$ are cospectral, and there exists a polynomial $p(x)$ such that $p(A) e_{u}=e_{v}$.

(ii) The vertices $u$ and $v$ are strongly cospectral.

Moreover, if $u$ and $v$ are cospectral, then any polynomial satisfying $p(A) e_{u}=e_{v}$ is such that $p(A) e_{v}=e_{u}$ and $p\left(\theta_{r}\right)= \pm 1$ for all $\theta_{r} \in \Phi_{u}$.

Proof. As we observed in Section 2, strongly cospectral vertices are cospectral. Thus the implication (ii) $\Longrightarrow$ (i) follows from the first lines of the proof of Lemma 6 . To see the converse, let $p(x)$ be a polynomial satisfying $p(A) e_{u}=e_{v}$. Because $p(A)$ is a symmetric matrix, it follows that $\left(p(A)^{2}\right)_{u, u}=1$. Vertices $u$ and $v$ are cospectral, so Lemma 1 implies that $\left(p(A)^{2}\right)_{v, v}=1$. Thus $p(A) e_{v}$ is a unitary vector, but $p(A)_{u, v}=1$, implying that $p(A) e_{v}=e_{u}$. As a consequence, $p(A)^{2} e_{u}=e_{u}$, and so if $\theta_{r} \in \Phi_{u}$, it follows that $p\left(\theta_{r}\right)= \pm 1$. This shows that $u$ and $v$ are strongly cospectral.

Here we introduce a definition. We say that $u$ and $v$ are antipodal ${ }^{1}$ vertices if

- there is a pseudo equitable partition which is simultaneously the distance partition of $u$ and $v$,

- $\{u\}$ and $\{v\}$ are singletons in this partition at maximum distance from each other,

- $u$ and $v$ are cospectral.

As pointed out by the anonymous referee, it is worth noting that if the distance partition of $u$ is pseudo equitable and $v$ is the only vertex at maximum distance, then the distance partition of $v$ is precisely the same partition, and therefore also pseudo equitable.

If the graph is regular, then a pair $u$ and $v$ of antipodal vertices is cospectral if and only if the parameters of the distance partition of $u$ are equal to the parameters of the distance partition of $v$. This is a consequence of Lemma 1 and of the fact that the number of closed walks of any length on vertices whose distance partition is equitable is determined by the parameters of the partition.

Theorem 8. If $u$ and $v$ are antipodal vertices in $X$, then $u$ and $v$ are spectrally extremal vertices and they are strongly cospectral. On the other hand, if $u$ is spectrally extremal, $u$ and $v$ are strongly cospectral, and their distance is equal to their eccentricity, then $u$ and $v$ are antipodal vertices.

\footnotetext{
${ }^{1}$ The name antipodal has been used in different contexts. We are consistent with at least one its uses, namely, if a regular graph can be partitioned into pairs of antipodal vertices, then it is an antipodal distance regular graph.
} 
Proof. If $u$ and $v$ are antipodal, then the weaker direction of Theorem 3 implies that $u$ is spectrally extremal and that there is a polynomial $p(x)$ such that

$$
p(A) e_{u}=e_{v} .
$$

From Lemma 7, we have that $u$ and $v$ are strongly cospectral.

The converse is an immediate consequence of Theorem 3 and the fact that strongly cospectral vertices are cospectral.

M. A. Fiol pointed out in a private communication that the condition on the theorem above that requires $u$ and $v$ to be at maximal distance cannot be dropped. In fact, the path on five vertices is an example of a graph containing a pair of spectrally extremal strongly cospectral vertices which are not at maximal distance from each other.

Note however that Lemma 6 implies that $v$ is a cut-vertex of $X$ unless $v$ is at maximal distance from $u$. So if $X$ is 2-connected, then it follows that any pair of spectrally extremal strongly cospectral vertices must be at maximal distance from each other.

The following lemma gives some interesting consequences.

Lemma 9. Suppose $u$ is a spectrally extremal vertex of $X$, and suppose $u$ and $v$ are strongly cospectral. Let $p(x)$ be the polynomial satisfying $p(A) e_{u}=e_{v}$, with $p\left(\theta_{r}\right)=\sigma_{r} \in$ $\{+1,-1\}$ for all $\theta_{r} \in \Phi_{u}$. Let $X^{\prime}$ be the component of $X \backslash v$ containing $u$, and $A^{\prime}=A\left(X^{\prime}\right)$. Then $p(x)$ satisfies $p\left(A^{\prime}\right) e_{u}=0$, that is, $p(x)$ is the minimal polynomial with respect to $u$ in $X^{\prime}$ (up to a constant).

Proof. Let $d(u, v)=g$. From Lemma 6 (i), we have that $v$ is the unique vertex at distance $g$ from $u$. Note that walks of length $g$ in $X$ pass by $v$ only if $v$ is its final vertex, so the entries of $p(A) e_{u}$ relative to vertices at distance at most $g-1$ from $u$ are equal to the respective entries of $p\left(A^{\prime}\right) e_{u}$, thus $p\left(A^{\prime}\right) e_{u}=0$. Because the eccentricity of $u$ in $X^{\prime}$ is $g-1$ and $p(x)$ has degree $g$, it follows that it is the minimal polynomial up to a constant.

Corollary 10. Let $u, v \in V(X)$. Suppose $\Phi_{u}=\left\{\theta_{0}, \ldots, \theta_{d^{*}}\right\}$, ordered in such way that $\theta_{r}>\theta_{r+1}$ for all $r$. If $u$ and $v$ are spectrally extremal and strongly cospectral, and $p(x)$ is such that $p(A) e_{u}=e_{v}$, then there is no index $r \in\left\{0, \ldots, d^{*}-2\right\}$ such that

$$
p\left(\theta_{r}\right)=p\left(\theta_{r+1}\right)=p\left(\theta_{r+2}\right) .
$$

Proof. Suppose otherwise that there is such index, say s. From Lemma 9, the roots of $p(x)$ are the eigenvalues of $X \backslash v$ in the support of $u$. Interlacing (see [4, Theorem 2.5.1]) implies that there are no two roots of $p(x)$ between $\theta_{r}$ and $\theta_{r+1}$ for any $r$, hence $p\left(\theta_{s}\right)=p\left(\theta_{s+1}\right)=p\left(\theta_{s+2}\right)$ implies that there are three roots of $\frac{d}{d x} p(x)$ between two of its real roots. This is a contradiction to the fact that all roots of $p(x)$ are real.

If we know that the pair of strongly cospectral vertices is at maximal distance, we can actually determine the values of $p\left(\theta_{r}\right)$ for all $r$. 
Theorem 11. Let $u, v \in V(X)$. Suppose $\Phi_{u}=\left\{\theta_{0}, \ldots, \theta_{d^{*}}\right\}$, ordered in such way that $\theta_{r}>\theta_{r+1}$ for all $r$. Then $u$ and $v$ are antipodal if and only if, for all $r \in\left\{0, \ldots, d^{*}\right\}$,

$$
E_{r} e_{v}=(-1)^{r} E_{r} e_{u}
$$

Proof. Let $d=d(u, v)$, and $p(x)$ the polynomial of degree $d$ such that $p(A) e_{u}=e_{v}$. If $p(x)$ is such that $p\left(\theta_{r}\right)=(-1)^{r}$, then $p(x)$ has at least $d^{*}$ roots, so $d \geqslant d^{*}$, and hence it could only be that $d=d^{*}$. So $u$ and $v$ are spectrally extremal, strongly cospectral, and at maximal distance. It follows from Theorem 8 that they are a pair of antipodal vertices.

For the converse, let $p(x)$ be the polynomial satisfying $p\left(\theta_{r}\right)=\sigma_{r} \in\{+1,-1\}$ with $p(A) e_{u}=e_{v}$. The existence of such $p(x)$ is implied in Theorem 8. Let $q(x)$ be the polynomial of minimal degree that satisfies $q\left(\theta_{r}\right)=(-1)^{r}$ for $r \in\left\{0, \ldots, d^{*}\right\}$. Therefore

$$
\begin{aligned}
1 & \geqslant\left|q(A)_{u, v}\right| \\
& =\left|\sum_{r=0}^{d^{*}}(-1)^{r} \prod_{s \neq r} \frac{1}{\theta_{r}-\theta_{s}}\right|\left(A^{d^{*}}\right)_{u, v} \\
& =\left(\sum_{r=0}^{d^{*}}(-1)^{r} \prod_{s \neq r} \frac{1}{\theta_{r}-\theta_{s}}\right)\left(A^{d^{*}}\right)_{u, v}, \quad \text { because all terms are positive, } \\
& \geqslant\left(\sum_{r=0}^{d^{*}} \sigma_{r} \prod_{s \neq r} \frac{1}{\theta_{r}-\theta_{s}}\right)\left(A^{d^{*}}\right)_{u, v} \\
& =p(A)_{u, v} \\
& =1 .
\end{aligned}
$$

Note that equality holds throughout if and only if $\sigma_{r}=(-1)^{r}$, as we wanted.

If a graph $X$ has diameter $d$, then Equation 1 implies that $X$ has at least $d+1$ distinct eigenvalues. We say that $X$ is spectrally extremal if equality holds. Note that every spectrally extremal graph contains at least one pair of spectrally extremal vertices. As pointed out by the anonymous referee, the ideas in the proof of Theorem 11 are not new, and in fact the result was known for spectrally extremal regular graphs (see [12]). The results below, however, only work for this special case.

Theorem 12 ([12], Theorem 4.3). Suppose $X$ is a spectrally extremal regular graph on $n$ vertices of diameter $d$, and that its distinct eigenvalues are $\theta_{0}>\ldots>\theta_{d}$. Suppose $u$ and $v$ are vertices at distance $d$. Then $u$ and $v$ are antipodal if and only if

$$
n \prod_{s=0}^{d} \frac{1}{\theta_{0}-\theta_{s}}=\sum_{r=0}^{d}(-1)^{r} \prod_{s \neq r} \frac{1}{\theta_{r}-\theta_{s}} .
$$

Proof. Let $p(x)$ be a polynomial such that $p(A)=E_{0}$, and let $J$ denote the all 1 s matrix. Because the graph is regular $E_{0}=(1 / n) J$, and so if $p(x)=a_{d} x^{d}+\ldots+a_{0}$, it follows that

$$
\left(A^{d}\right)_{u, v}=\frac{1}{n}\left(\prod_{s=0}^{d} \frac{1}{\theta_{0}-\theta_{s}}\right)^{-1}
$$


for all vertices $u$ and $v$ at distance $d$. The result now follows from Theorem 11 .

Corollary 13 ([12], Theorem 4.4). Suppose $X$ is a spectrally extremal regular graph on $n$ vertices of diameter $d$. If the eccentricity of every vertex is $d$ and if

$$
n \prod_{s=0}^{d} \frac{1}{\theta_{0}-\theta_{s}}=\sum_{r=0}^{d}(-1)^{r} \prod_{s \neq r} \frac{1}{\theta_{r}-\theta_{s}},
$$

then $X$ is an antipodal distance regular graph.

Proof. It follows from Theorem 2 and Theorem 12.

\section{State transfer on spectrally extremal graphs}

In this section, we relate the work in the past section to state transfer.

Theorem 14. Suppose $u$ is a spectrally extremal vertex of $X$, and $v$ is a vertex at maximal distance from $u$. Let $\Phi_{u}=\left\{\theta_{0}, \ldots, \theta_{d^{*}}\right\}$, where, for some integers $a, \Delta$ and $b_{r}$ with $r=0, \ldots, d^{*}$, we have $\theta_{r}=\frac{a+b_{r} \sqrt{\Delta}}{2}$ satisfying $\theta_{r}>\theta_{r+1}$. Then $X$ admits perfect state transfer between vertices $u$ and $v$ if and if only if the following conditions hold.

(i) The vertices $u$ and $v$ are antipodal.

(ii) If $r$ is odd, then the powers of two appearing in the factorization of each of the differences $b_{0}-b_{r}$ are constant, let us say $\alpha$.

(iii) If $r$ is even, then the power of two in the factorization of each $b_{0}-b_{r}$ is larger than $\alpha$.

If the conditions hold, perfect state transfer happens at time $\frac{\pi}{2^{\alpha}}$ (and any of its odd multiples).

Proof. From Lemma 5, perfect state transfer implies strong cospectrality. The vertices are at maximal distance from each other, and if they are strongly cospectral, Theorem 8 says that they are antipodal. Note that

$$
U(t) e_{u}=\sum_{r=0}^{d^{*}} \mathrm{e}^{\mathrm{i} t \theta_{r}} E_{r} e_{u} .
$$

Lemma 11 implies that

$$
e_{u}=\sum_{r=0}^{d^{*}}(-1)^{r} E_{r} e_{v},
$$

therefore perfect state transfer is now equivalent to existing a time $t$ such that

$$
\frac{\mathrm{e}^{\mathrm{i} t \theta_{0}}}{\mathrm{e}^{\mathrm{i} t \theta_{r}}}=(-1)^{r}
$$


and this is equivalent to

$$
t\left(\theta_{0}-\theta_{r}\right)=k_{r} \pi,
$$

where $k_{r}$ is an integer with the same parity as $r$. This condition is equivalent to (ii) and (iii), and also gives the expression for the time.

Corollary 15. Suppose $X$ is a spectrally extremal graph of diameter $d$ and eigenvalues $\theta_{0}>\ldots>\theta_{d}$. Suppose $X$ admits perfect state transfer at time $\tau$ between vertices $u$ and $v$ at distance $d$. If $d(z, w)=d$, then $\left(A^{d}\right)_{z, w} \leqslant\left(A^{d}\right)_{u, v}$, and equality happens if and only if $X$ admits perfect state transfer between $z$ and $w$ at time $\tau$.

Proof. The inequality is a straightforward application of Lemma 6 (ii). If equality happens, then Lemma 6 (ii) says that $z$ and $w$ are strongly cospectral. They are at maximum distance, so by Theorem 8, they are antipodal. Combining this with Theorem 14 and the fact that the eigenvalue support of $z$ and $w$ is equal to the eigenvalue support of $u$ and $v$, it follows that perfect state transfer between $z$ and $w$ happens at time $\tau$.

In the case where $X$ is a regular graph, we can say more.

Corollary 16. Suppose $X$ is a spectrally extremal regular graph on $n$ vertices of diameter $d$, distinct eigenvalues $\theta_{0}>\ldots>\theta_{d}$. Then $X$ admits perfect state transfer between any pair $(u, v)$ of vertices at distance $d$ if and only if

(i) All eigenvalues are integers.

(ii) If $r$ is odd, then the powers of two appearing in the factorization of each of the differences $\theta_{0}-\theta_{r}$ are constant, let us say $\alpha$.

(iii) If $r$ is even, then the power of two in the factorization of $\theta_{0}-\theta_{r}$ is larger than $\alpha$.

(iv) The following equality holds

$$
n \prod_{s=0}^{d} \frac{1}{\theta_{0}-\theta_{s}}=\sum_{r=0}^{d}(-1)^{r} \prod_{s \neq r} \frac{1}{\theta_{r}-\theta_{s}} .
$$

Proof. Because the graph is spectrally extremal, all eigenvalues are in the eigenvalue support of the vertices at distance $d$. Note that the largest eigenvalue is integer, so Theorem 4 implies that all eigenvalues are integers. Thus it follows from Theorems 12 and 14 that the other conditions are equivalent to perfect state transfer between vertices at distance $d$.

The following is an immediate consequence of the result above and Corollary 13.

Corollary 17. If $X$ is a spectrally extremal regular graph of diameter $d$ in which the eccentricity of every vertex is $d$, and if perfect state transfer happens between any pair of vertices at distance $d$, then $X$ is a distance-regular graph.

We finally observe that the result above is a good example on how the existence of perfect state transfer can imply deep structural properties in a graph. 


\section{$5 \quad$ Examples}

\section{Cartesian powers of $P_{3}$ :}

It was shown in [6] that if $X$ admits perfect state transfer, then any Cartesian power of $X$ does so as well. In [11], we learned that $P_{3}$ and its Cartesian powers provide examples of non-regular graphs with pairs of antipodal vertices. Therefore $P_{3}$ and its Cartesian powers are graphs satisfying Theorem 14. As an illustration, we depict below $P_{3} \square P_{3}$, and the highlighted vertices are involved in perfect state transfer.

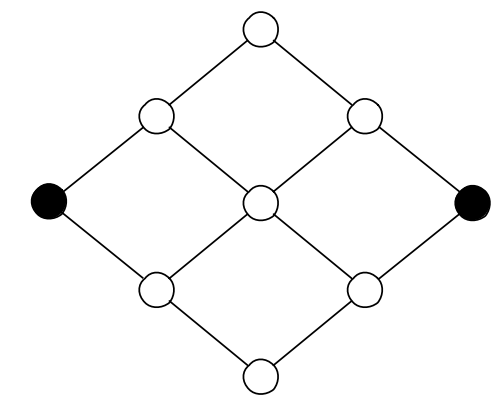

The distance partition of the black vertices is pseudo equitable, the vertices are strongly cospectral at maximal distance, and the eigenvalues of the graph are

$$
\{2 \sqrt{2}, \sqrt{2}, 0,-\sqrt{2},-2 \sqrt{2}\} .
$$

Altogether, this implies that perfect state transfer occurs between the black vertices at time $\pi / \sqrt{2}$.

\section{Graphs derived from hypercubes:}

It is well known (see [6]) that hypercubes of any dimension admit perfect state transfer between antipodal vertices. These are regular graphs - in fact, distance-regular graphs. However it is possible to perturb a hypercube in such a way that the resulting graph is no longer regular, but contains a pair of antipodal vertices whose distance partition is pseudo equitable with coinciding parameters to those of the partition in the original hypercube, and therefore perfect state transfer still occurs. The example below depicts the 4-dimensional hypercube and one of such variations.
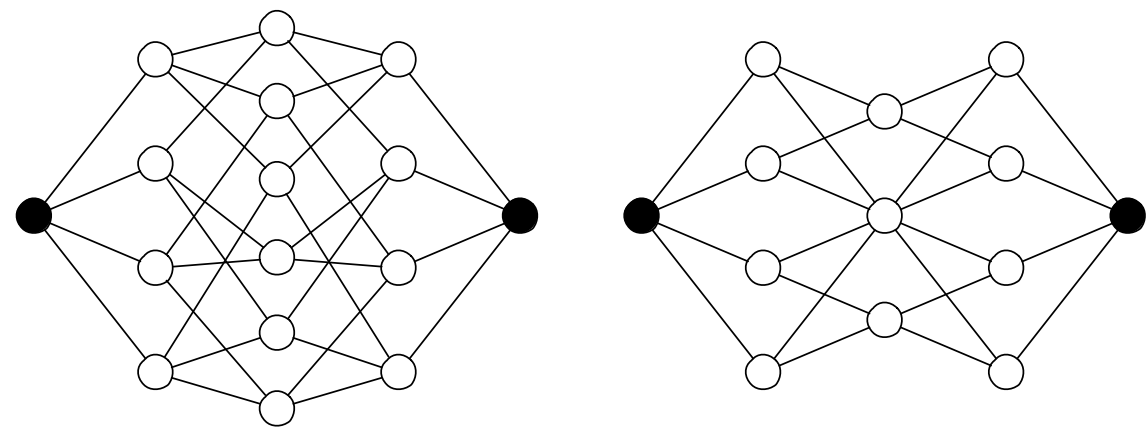
The graph $X$ on the right hand side of the picture is not regular, but the black vertices are antipodal, and their pseudo equitable distance partition has the same parameters as the distance partitions of the hypercube. Hence the eigenvalues in their support are $\{4,2,0,-2,-4\}$, and therefore, by Theorem 14, the graph $X$ admits perfect state transfer at time $\pi / 2$. Iterated Cartesian powers of $X$ will present similar features. To the best of our knowledge, this infinite family of graphs admitting perfect state transfer has not appeared in the literature.

As a special feature of this example, we note that $X \square K_{2}$ is a simple graph admitting perfect state transfer between vertices at distance five, and among all known such graphs, this is the one with the smallest number of vertices and edges. More generally, we are improving the known trade-off between the size of the graph and the distance between the vertices involved in perfect state transfer for all odd distances, which was previously achieved by hypercubes in odd dimension.

\section{Acknowledgement}

I'd like to acknowledge Chris Godsil for very fruitful discussions about perfect state transfer and more specifically about the results in this paper. I also acknowledge M. A. Fiol, who was visiting Waterloo during the end of my $\mathrm{PhD}$ and with whom I discussed the subject of spectrally extremal graphs. Finally, I am thankful to the anonymous referee for the valuable comments and for pointing out that some results in Section 3 were published in $[12]$.

\section{References}

[1] Ricardo Javier Angeles-Canul, Rachael M Norton, Michael C Opperman, Christopher C. Paribello, Matthew C. Russell, and Christino Tamon. Perfect state transfer, integral circulants, and join of graphs. Quantum Information \& Computation, 10(34):325-342, 2010.

[2] Rachel Bachman, Eric Fredette, Jessica Fuller, Michael Landry, Michael Opperman, Christino Tamon, and Andrew Tollefson. Perfect state transfer on quotient graphs. Quantum Information \& Computation, 12(3-4):293-313, 2012.

[3] Norman Biggs. Algebraic Graph Theory. Cambridge University Press, Cambirdge, 1993.

[4] Andries E. Brouwer and Willem H. Haemers. Spectra of Graphs. Universitext. Springer, New York, 2012.

[5] Wang-Chi Cheung and Chris D. Godsil. Perfect state transfer in cubelike graphs. Linear Algebra and its Applications, 435(10):2468-2474, 2011.

[6] Matthias Christandl, Nilanjana Datta, Tony C. Dorlas, Artur Ekert, Alastair Kay, and Andrew J Landahl. Perfect transfer of arbitrary states in quantum spin networks. Physical Review A, 71(3):32312, 2005. 
[7] Matthias Christandl, Nilanjana Datta, Artur Ekert, and Andrew J. Landahl. Perfect State Transfer in Quantum Spin Networks. Physical Review Letters, 92(18):187902, 2004.

[8] Gabriel Coutinho, Chris D. Godsil, Krystal Guo, and Frédéric Vanhove. Perfect state transfer on distance-regular graphs and association schemes. Linear Algebra and its Applications, 478(0):108-130, 2015.

[9] Cristina Dalfó, Edwin R. van Dam, Miquel A. Fiol, Ernest Garriga, and Bram L. Gorissen. On almost distance-regular graphs. Journal of Combinatorial Theory, Series A, 118(3):1094-1113, 2011.

[10] Miquel A. Fiol and Ernest Garriga. From local adjacency polynomials to locally pseudo-distance-regular graphs. Journal of Combinatorial Theory, Series B, 71(2):162-183, 1997.

[11] Miquel A. Fiol, Ernest Garriga, and José L A. Yebra. Locally pseudo-distance-regular graphs. Journal of Combinatorial Theory, Series B, 68(2):179-205, 1996.

[12] Miquel A. Fiol, Ernest Garriga, and José L A. Yebra. From regular boundary graphs to antipodal distance-regular graphs. Journal of Graph Theory, 27(3):123-140, March 1998.

[13] Chris D. Godsil. Algebraic Combinatorics. Chapman \& Hall, New York, 1993.

[14] Chris D. Godsil. State transfer on graphs. Discrete Mathematics, 312(1):129-147, 2012.

[15] Chris D. Godsil. When can perfect state transfer occur? Electronic Journal of Linear Algebra, 23:877-890, 2012.

[16] Chris D. Godsil and John Shawe-Taylor. Distance-regularised graphs are distanceregular or distance-biregular. Journal of Combinatorial Theory, Series B, 43(1):14$24,1987$.

[17] Vivien M. Kendon and Christino Tamon. Perfect State Transfer in Quantum Walks on Graphs. Journal of Computational and Theoretical Nanoscience, 8(3):422-433, 2011.

[18] Guang-Siang Lee and Chih-wen Weng. A spectral excess theorem for nonregular graphs. Journal of Combinatorial Theory, Series A, 119(7):1427-1431, 2012.

[19] Marko D. Petković and Milan Bašić. Further results on the perfect state transfer in integral circulant graphs. Computers \& Mathematics with Applications, 61(2):300$312,2011$.

[20] Edwin R. van Dam. The spectral excess theorem for distance-regular graphs: a global (over)view. Electronic Journal of Combinatorics, 15(1):\#R129, 10, 2008.

[21] Jiang Zhou, Changjiang Bu, and Jihong Shen. Some results for the periodicity and perfect state transfer. Electronic Journal of Combinatorics, 18(1):\#P184, 7, 2011. 